\title{
Lay people are unimpressed by the effect sizes typically reported in psychological science
}

\author{
Jonathon McPhetres ${ }^{1,2, *} \&$ Gordon Pennycook ${ }^{2}$
}

${ }^{1}$ Massachusetts Institute of Technology, Sloan School
${ }^{2}$ University of Regina, Hill/Levene Schools of Business
${ }^{*}$ Corresponding author: jon.mcphetres@gmail.com

Word count: 3,536

\begin{abstract}
It is recommended that researchers report effect sizes along with statistical results to aid in interpreting the magnitude of results. According to recent surveys of published research, psychologists typically find effect sizes ranging from $r=.11$ to $r=.30$. While these numbers may be informative for scientists, no research has examined how lay people perceive the range of effect sizes typically reported in psychological research. In two studies, we showed online participants $(N=1,204)$ graphs depicting a range of effect sizes in different formats. We demonstrate that lay people perceive psychological effects to be small, rather meaningless, and unconvincing. Even the largest effects we examined (corresponding to a Cohen's $d=.90$ ), which are exceedingly uncommon in reality, were considered small-to-moderate in size by lay people. Science communicators and policymakers should consider this obstacle when attempting to communicate the effectiveness of research results.

Keywords: effect size; science communication; policy; public understanding of science.
\end{abstract}

**This working paper has not yet been peer-reviewed ** 
Although the reporting of effect sizes has been recommended for over two decades (Cohen, 1992; Wilkinson \& American Psychological Association Task Force on Statistical Inference, 1999), they are only now becoming commonplace in statistical reporting. An effect size exists as a descriptive indicator of the effectiveness of a treatment or the strength of an association between two variables. However, these effect sizes exist mainly for the benefit of scientists and it is unknown how the public perceives effect sizes.

Initially, Cohen (Cohen, 1992) suggested arbitrary rules of thumb to determine whether an effect size was small $(d=.20)$, medium $(d=.50)$, or large $(d=.80)$. He determined these rules visually, by simply looking at graphs and determining what a reasonable effect should look like. However, recent research suggests that the effect sizes typically found by social scientists are much smaller than initial recommendations by Cohen. Meta-analyses of social psychological research, at least, find effects ranging from $r=.10$ to $r=.30$ (Gignac \& Szodorai, 2016a) suggesting that scientists should rethink what is considered small, medium and large in the context of psychological research (Funder \& Ozer, 2019). This information has important implications for researchers because it means the effect size one will find is probably smaller than the effect they would prefer, at least visually. However, scientists have statistical training, they think of effect sizes numerically (rather than visually), and they have guidelines to determine what effect size they should expect. Lay people do not have this benefit and, instead, probably approach research results more like Cohen did when he decided what a medium effect would look like.

Thus, the range of effect sizes found in the social sciences has important implications for those wishing to communicate their findings to the general public. Scientists and policymakers often attempt to communicate information about the effectiveness of a treatment or policy in 
order to garner support. However, communicating evidence for the effectiveness of health policies is often ineffective. For example, providing the public with quantitative information about treatment results is not always effective in generating support for a policy (Reynolds, Pilling, \& Marteau, 2018) and, when it is statistically significant, these effects are very small (Reynolds et al., 2018; Reynolds, Stautz, Pilling, van der Linden, \& Marteau, 2020). This information may be surprising when viewed through the eyes of a scientist because seemingly "small" effects may be impactful when applied to the larger population.

One possible issue, therefore, is that the effect sizes generally seen as large and/or meaningful to scientists may not be compelling to lay people. A further issue is that the data visualization techniques employed by scientists to communicate uncertainty (e.g. error bars, depictions of variance) may not be easily understood by those without statistical training (Hofman, Goldstein, \& Hullman, 2020). Importantly, effects that appear visually large-for example, a mean difference with a large standard deviation — may be misleading to the public. In contrast, effects estimated with high precision (i.e., a small standard deviation) may actually be interpreted with more confidence by a scientist, but would appear small when represented visually.

In two studies, we sought to examine how lay people perceive the effect sizes commonly found in the social sciences. We presented subjects with graphs depicting effects in various ways and asked subjects to rate them on different dimensions. Our studies were preregistered prior to data collection. Data, materials, and preregistration plans can be found at the following link: https://osf.io/cv7uw/. 


\section{Study 1}

Study 1 was conducted as an initial investigation in to how the public perceived effect sizes. We showed participants depictions of effect sizes using images of two overlapping normal distributions of data. The sample size, materials, and analysis procedures were preregistered prior to data collection: https://osf.io/3u62b.

\section{Participants, Materials, and Procedure}

Subjects $(N=604)$ were recruited through Amazon's Mechanical Turk. Complete demographics are displayed in Table S1. Subjects accessed an online survey hosted on the Qualtrics platform. Subjects first received a brief introduction into the concepts of effect sizes and the normal distribution of data. They then viewed a graph depicting normally distributed data and responded to a series of questions about the graph, and then completed demographic questions.

The introduction depicted the hypothetical distribution of women's heights with arrows identifying key points of the graph (i.e., the mean, the x-axis, the y-axis) to aid in interpretation. We did not depict the comparison of men and women's heights so as to avoid influencing subjects' perceptions of effect sizes on the coming task. Subjects were then asked if they understood the previous information (Yes/Somewhat/No).

Subjects were then randomised into one of four conditions where they viewed an image of two overlapping distributions corresponding to an effect size of Cohen's $d=.20(n=160), d=$ $.40(n=145), d=.60(n=159), d=.90(n=140)$ and then answered 6 questions in randomized order. The distributions were generic, labelled as "Group A" and "Group B" and there were no numeric indicators so as to avoid influencing perceptions in any way. Participants rated the image on the following dimensions. 
Size of the effect. Participants rated the size of the effect on a scale from 1 (There is NO effect) to 5 (There is a VERY LARGE effect).

Meaningfulness of the effect. Participants rated the meaningfulness of the effect on a scale from 1 (not meaningful at all) to 7 (extremely meaningful).

Making predictions from the data. Subjects were asked to make a prediction using the information in the graph: "If you were to choose a single person from each group at random, what would be the most likely outcome?" Options were The person from Group A would certainly have a higher score (5); It's only slightly likely that the person from Group A would have a higher score (4); The two people would have the same (or very similar) scores (3); It's only slightly likely that the person from Group B would have a higher score (2); and The person from Group B would certainly have a higher score (1).

Success of the experiment. Subjects were asked "If this were an experiment where you hoped to find differences between the two groups, would you say the experiment was a success?" Options were Yes or No.

Grant funding allocation. Subjects were asked "If you were on a committee in charge of grant funding, would you decide to allocate more grant funds to this research project?" Options were Yes or No.

Definitiveness of the result. Subjects were asked "Would you interpret this data as showing that Group A definitively has higher scores than Group B?" Options were Yes or No.

\section{Results}

Only 6 participants indicated that they did not understand the descriptions of the distribution graphs; thus, we continued on with our analyses as planned. Zero-order correlations are presented in the supplementary materials (see Table S2). 
A series of means comparisons indicates that people only slightly distinguished between the different effect sizes (see Figure 1). Larger effect sizes were seen as somewhat larger, F(3, $596)=3.01, \mathrm{p}=.030$, eta $=.015$, and slightly more meaningful, $\mathrm{F}(3,596)=3.45, \mathrm{p}=.016$, eta $=$ .017 , than smaller effect sizes. Effect size condition did not influence people's predictions about whether one person chosen at random from each group would have different scores $\mathrm{F}(3,596)=$ $1.03, \mathrm{p}=.38$, eta $=.005$. Simple comparisons indicate that the largest perceived differences were when between effect sizes $d=.20$ and $d=.90$ (i.e. a difference of .70 ). Table 1 shows the Cohen's d effect size comparisons between all conditions.

Analysis of dichotomous outcomes (see Figure 1) revealed that around half of all participants agreed with each statement; this percentage was only slightly greater in the larger effect size conditions. Participants were slightly more likely to agree that the experiment was a success when the effect size was $d=.60$ or $d=.90, \chi^{2}(3)=10.23, p=.017, V=.092$. However, effect size condition did not influence participants allocation of grant funds, $\chi^{2}(3)=4.90, p=.18$, $V=.063$, or their belief that Group A is definitely higher than Group $\mathrm{B}, \chi^{2}(3)=1.98, \mathrm{p}=.58, V=$ .041 .

\section{Discussion}

In summary, Study 1 demonstrates that people generally view the range of effect sizes in social psychology as being small and only moderately meaningful. Participants did distinguish somewhat between the different effect sizes depicted here-for example, a $d=.20$ was seen as smaller than a $d=.90$ - though even the largest effect was still rated as "small." Further, participants did not appear to be convinced by these effects: they did not make strong predictions from the data about whether a persons' score would be higher or lower and they did not see the results as definitively showing that one group had higher scores. 
Figure 1. Depiction of means, data distributions, and percentages for all variables in Study 1. The left panel depicts continuous outcomes and the diamonds indicate the mean; the right panel depicts dichotomous outcomes and the bars indicate percent agreement; Meaningful ratings range from 1 (not at all meaningful) to 7 (extremely meaningful); Prediction ratings range from 1 (The person from Group A would have higher scores) to 5 (The person from Group B would have higher scores); Size ratings range from 1 (There is no effect to 5 (The effect is very large).
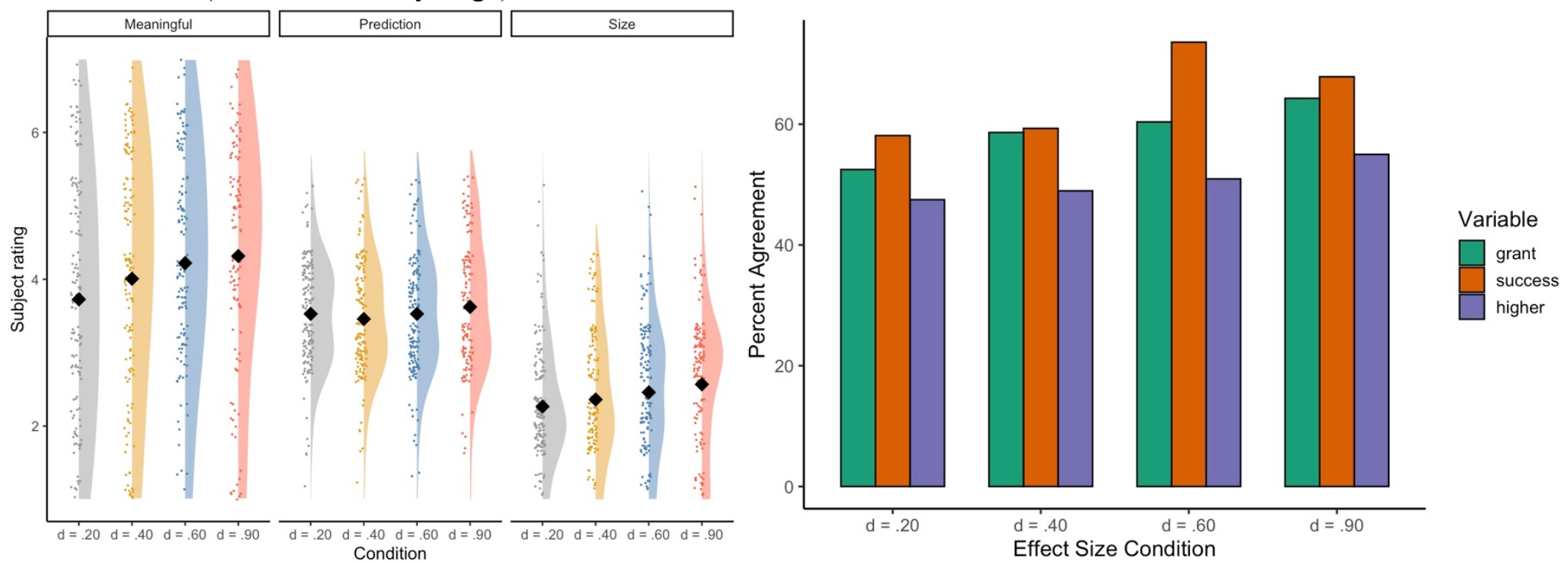

Table 1. Means, standard deviations, and Cohen's $\boldsymbol{d}$ effect sizes for multiple comparisons across conditions. M and SD refer to mean and standard deviation respectively; columns 1-3 represent Cohen's d for each comparison.

\begin{tabular}{|c|c|c|c|c|c|c|c|c|c|c|c|c|c|c|c|}
\hline \multirow[b]{2}{*}{ Condition } & \multicolumn{5}{|c|}{ Meaningfulness of effect } & \multicolumn{5}{|c|}{ Prediction } & \multicolumn{5}{|c|}{ Size of effect } \\
\hline & $M$ & $S D$ & 1 & 2 & 3 & $M$ & $S D$ & 1 & 2 & 3 & $M$ & $S D$ & 1 & 2 & 3 \\
\hline 1. $d=.20$ & 3.73 & 1.74 & & & & 3.53 & 0.70 & & & & 2.27 & 0.83 & & & \\
\hline 2. $d=.40$ & 4.01 & 1.72 & 0.16 & & & 3.46 & 0.77 & 0.09 & & & 2.36 & 0.90 & 0.11 & & \\
\hline 3. $d=.60$ & 4.22 & 1.70 & 0.29 & 0.12 & & 3.53 & 0.80 & 0.00 & 0.09 & & 2.46 & 0.94 & 0.21 & 0.11 & \\
\hline 4. $d=.90$ & 4.32 & 1.77 & 0.34 & 0.18 & 0.06 & 3.62 & 0.89 & 0.11 & 0.20 & 0.11 & 2.57 & 0.98 & 0.33 & 0.22 & 0.11 \\
\hline
\end{tabular}




\section{Study 2}

In Study 1, participants only saw graphs depicting the normal distribution. Study 2 was conducted to examine the effect of different graph types and different levels of variance. The sample size, materials, and analysis procedures were preregistered prior to data collection: https://osf.io/h5m8k.

\section{Participants and Materials}

Subjects for Study $2(N=600)$ were recruited through Prolific Academic (complete demographics are presented in Table S1). As with Study 1, subjects were first given an overview of each graph type. After each overview, subjects answered two items to test their understanding of each graph type before moving on to provide ratings for each of 9 graph images.

Subjects were randomized into one of four conditions where they saw graphs depicting data corresponding to Cohen's $d$ of either $.20(n=163), .40(n=130), .60(n=155)$, or $.90(n$ =152). Within each condition, subjects rated three types of graphs: bar graphs, violin graphs, or correlation graphs. The graphs types were presented in random order with the constraint that the correlation graph either came first or third. For each graph type, subjects also rated three versions of that graph depicting the same effect size (i.e. Cohen's $d=.20$ ) but at three different levels of data dispersion: $S D=.5$ (low variance), $S D=1.00$ (medium variance), and $S D=2.00$ (high variance). Thus, each participant rated 9 graphs in total in a 4 (between-subjects: effect size condition) x 3 (within-subjects: variance level) design; each graph type was analyzed separately.

The dependent variables were the same as in Study 1 with three exceptions. The item "Would you interpret this graph as showing that Group A is definitively higher than Group B" was worded incorrectly for the bar and violin graphs (the graphs depicted Group B as higher), so it is not interpreted here. For the correlation graphs, that item was replaced with a question 
asking whether "the two variables are definitely related." Finally, the item asking about a prediction for scores of a randomly chosen person from each group was not asked for the correlation graphs because it does not apply.

\section{Results}

Overall, very few subjects reported not understanding the introductions to the graphs (bar: 3\%; violin: 3.5\%; correlation: $2 \%$ ). Two item quizzes on the content of each graph type revealed that a majority of subjects answered both questions correctly (bar: 80\%; violin: $80 \%$; correlation: 78\%) suggesting they could read the graphs satisfactorily (see Figure S1). Zero-order correlations are reported in the supplementary materials (see Tables S3-5).

For simplicity, we report here an abridged set of analyses; the full set of analyses are reported in the supplementary materials (see Table S6-8 and Figures S2-3). Analyses for each graph type were carried out separately, as stated in our preregistration. For each dependent variable, we used a mixed-model including random intercepts for subject and effect size condition and variance conditions as predictors, along with their interaction. The interaction is not relevant to our investigation, so we do not focus on it here.

Did people perceive the effects as small or large? There were main effects of effect size condition (bar graph $\eta^{2}: .06$; violin graph $\eta^{2}: .02$; correlation $\eta^{2}: .02$ ) with larger effect sizes being perceived as slightly larger. Variance condition also had main effects (bar graph $\eta^{2}:$. 12; violin graph $\eta^{2} . .08$; correlation $\eta^{2} . .02$ ) with lower variance levels being associated with slightly greater perceived size. Full model statistics are available in Table S6.

Figure 2 displays Cohen's d effect sizes for multiple comparisons across effect size conditions. As can be seen, there were greater perceived differences between conditions as the difference between the effect size conditions grew larger. However, subjects still perceived even 
the largest effects $(\mathrm{d}=.90)$ in the 'small' to 'medium' range (see Figure S2 and Table S9). In fact, across the entire set of ratings, very few were effects perceived as being "large" $(10 \%)$ or "very large" (2\%) in size.

Did people perceive the effects as meaningful? Effect size condition influenced subjects' perceptions of the size of the effect for each graph type (bar graph $\eta^{2}$ : .06; violin graph $\eta^{2}: .02$; correlation graph $\eta^{2}: .02$ ). Variance condition also had main effects (bar graph $\eta^{2}: .14$; violin graph $\eta^{2}$ : .10; correlation graph $\left.\eta^{2}: .06\right)$. Full model statistics are available in Table S6.

Figure 3 depicts the data distributions for perceptions of the meaningfulness of each effect. In general, larger effect sizes were perceived as slightly more meaningful. For the bar and violin graphs, higher variance levels were associated with slightly greater meaningfulness compared to lower variance. However, for the correlation graphs, lower variance was associated with slightly greater meaningfulness.

How do people use the data? After viewing the bar and violin graphs, subjects were asked to make a prediction about the scores of two randomly selected subjects. There were main effects of effect size condition (bar graph $\eta^{2}: .04$; violin graph $\eta^{2}: .04$ ) and variance condition (bar graph $\eta^{2}: .06$; violin graph $\eta^{2}: .01$ ). Full model statistics are available in Table S6; data is depicted in Figure S2.

In general, subjects expected that a person randomly chosen from Group B would have a slightly higher score than a person chosen from Group A when the effect size was larger and variance was higher. However, these expectations were not very strong, as means hovered around the mid-points of the scales (e.g. indicating a response of "The two people would have the same (or very similar) scores"). This suggests that subjects perceived a great deal of overlap between the two hypothetical groups depicted in the figures. 
Does effect size influence grant funding? The mixed-model we preregistered would not converge, so we ran a fixed-effects model. There were main effects of effect size condition (Bar graph: $V=.19$; violin graph: $V=.13$; correlation graph: $V=.09)$ with grant funding being allocated to studies with larger effect sizes. There were also main effects of variance condition (Bar graph: $V=.10$; violin graph: $V=.10$; correlation graph: $V=.09$ ). For the bar and violin graphs, grant funding was allocated more often in medium and high variance conditions, but for the correlation graphs grant funding was allocated more in the low variance conditions. There were no interactions. These effects are depicted in Figure S3.

Did people consider the experiment a success? The mixed-models we preregistered would not converge, so we ran fixed-effects models. There were main effects of effect size condition (Bar graph: $V=.19$; violin graph: $V=.13$; correlation graph: $V=.10$ ) with larger effect sizes being perceived as successful. There were also main effects of variance condition (Bar graph: $V=.14$; violin graph: $V=.14$; correlation graph: $V=.11$ ) with higher more variance being considered more successful for the bar and violin graphs, but low variance being considered most successful for the correlation graphs. There were no interactions. Data is depicted in Figure S3.

Were the two variables related? Finally, for the correlation graphs only, subjects were asked if the two variables were related. The mixed-model we preregistered would not converge, so we ran a fixed-effects model. There was a main effect of condition (correlation graph: $V=$ .06) with greater effect sizes generally seen as somewhat more indicative of a relationship. There was also a main effect of variance (correlation graph: $V=.11$ ) with low variance conditions being seen as more indicative of a relationship. There was no interaction. Full data is depicted in Figure S3 and Table S7. 


\section{Discussion}

In Study 2, we extended our findings from Study 1 by presenting participants with a range of visualizations. We also expanded our experimental conditions by displaying a range of variance levels. Our findings largely echoed those of Study 1: participants generally saw all effects as small and relatively meaningless, and did not seem very convinced by the effects as depicted here.

However, one result that is particularly important to focus on: the variance level conditions accounted for more variance than did effect size condition. For the bar and violin graphs, higher variance levels were associated with slightly greater meaningfulness compared to lower variance. However, for the correlation graphs, lower variance was associated with slightly greater meaningfulness. This can suggest that when the raw data is depicted (as in a correlation scatter plot) people use this information to infer certainty. However, when it is depicted abstractly (as in the violin and bar graphs), people focus on the absolute mean differences.

Nonetheless, the participants exhibited the same pattern of inference as in Study 1. Even when focusing on the absolute mean differences, even the largest effects $(d=.90)$ were rated in the small-to-moderately meaningful range, and as being small-to-moderate in size. This still suggests that, no matter the visualization type, the absolute effect size itself is still more influential than the variety of visualization techniques investigated here. 
Figure 2. Effect sizes for simple comparisons between conditions in Study 2. The dependent variable is "What size would you say this effect is?"; Diamonds depict the standardized mean difference between experimental conditions (e.g. simple comparisons) based on effect size

difference and variance level; the widest point of the diamond indicates the observed mean difference and the ends of the diamonds indicate $95 \%$ confidence intervals.
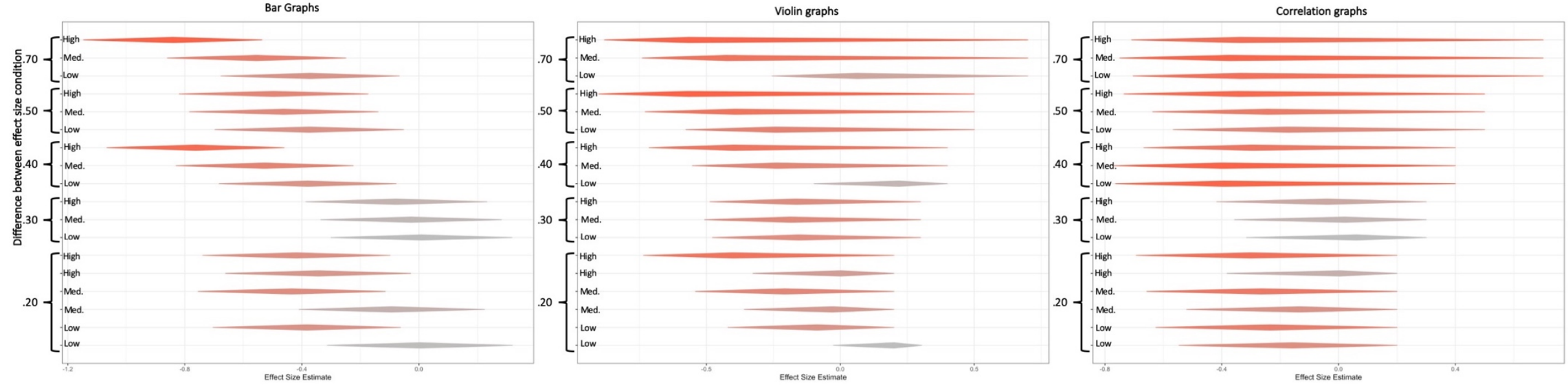

Figure 3. Depiction of raw data and mean by effect size condition and variance condition in Study 2. Solid shapes indicate density at each region; solid lines depict means within each condition.
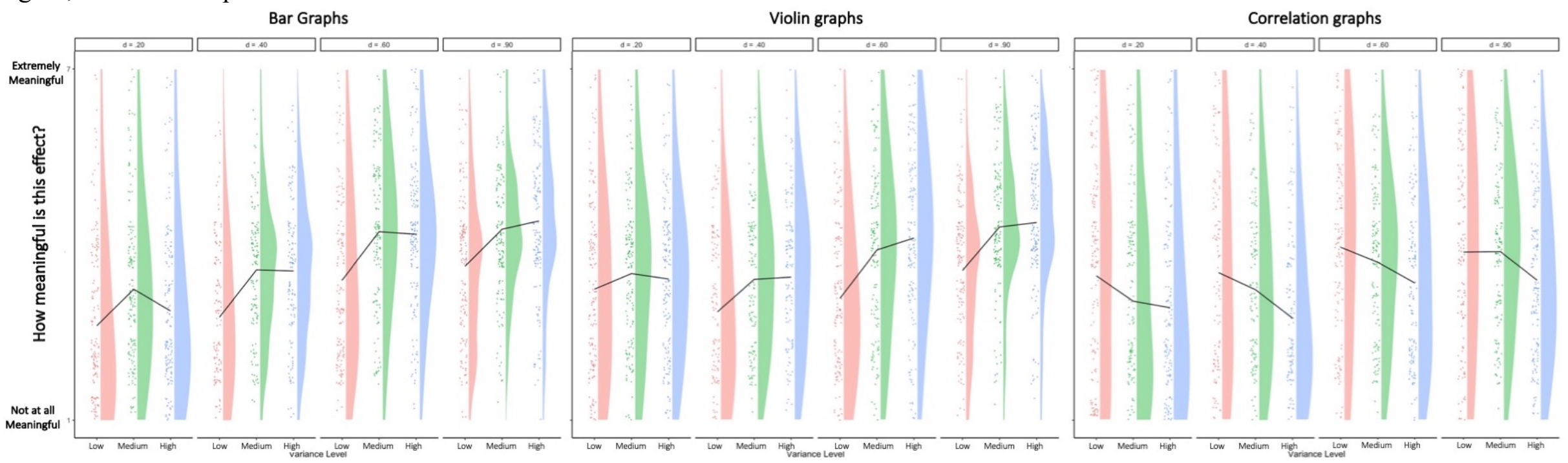


\section{General Discussion}

In two studies, subjects viewed graphs depicting effect sizes that are commonly found in psychological research, perceiving them to be small, rather meaningless, and unconvincing. However, graphs which depicted the raw data - correlation graphs - may be the most effective in communicating uncertainty, because greater data dispersion was associated with lower estimates of effect magnitude. These results suggest that, while scientists may have an understanding of what effects sizes may be reasonably expected, communicating this information to the general public may be a difficult task.

There are a few things to notice about the data presented in Figures 2 and 3. First, the stark comparisons between effect size conditions were attenuated as the complexity of the graphs increased: large differences were observed between effect sizes when data were depicted via bar graphs, but the differences between effect size conditions were less apparent for the correlation graphs. Thus, the range of effect sizes within which social psychologists put great emphasis do not seem to be very influential for lay people. However, response patterns and distributions for each graph type are remarkably similar and did not diverge from one another in a notable fashion (see Figure S2).

Second, subjects still viewed even the largest effect sizes $(d=.90)$ as quite small (see Figure S2) and meaningless (see Figure 3). On average, subjects estimated the size of the effect to be in the "no effect" to "small effect" range. Estimates only broke the mid-point for the meaningfulness ratings (i.e. 'somewhat meaningful) on violin graphs in the $d=.60$ and $d=.90$ conditions (see Tables S9-11 for complete means). Thus, even the effect sizes that social psychologists would consider 'very large' (e.g. $d=.90)$ and account for less than $3 \%$ of all 
reported effects in individual differences research (Gignac \& Szodorai, 2016b) still do not seem very compelling to lay people.

Finally, the level of data dispersion was more influential in how subjects interpreted the graphs: variance condition accounted for nearly twice the variance as effect size condition on average (see Table S6). This is possibly because greater variance requires a larger mean difference in order to achieve the same effect size. Thus, it appears that people gravitate towards interpreting the mean difference rather than accounting for the precision with which a given mean is estimated. This mechanism is apparent especially when considering that variance was positively associated with size estimates and meaningfulness ratings for the bar and violin graphs, but negatively associated for the correlation graphs. This mechanism is again apparent when considering how people made predictions about the scores of two random people: people were not strongly convinced that a random person from Group B would have higher scores than a random person from Group A.

\section{Conclusions}

Communicating scientific results is an important task for researchers. Namely, the ability to get the public on board with policy changes, laws, or medical treatments requires that information is convincingly communicated. Past research has found this to be a difficult task (Reynolds et al., 2018). The present results would suggest that this was difficult because lay people are likely not compelled by the effect sizes typically found in social sciences. Thus, a task

for researchers, policymakers, and science communicators is to determine how to effectively and accurately communicate scientific results to the public with this difficulty in mind. 


\section{References}

Cohen, J. (1992). A Power Primer. Psychological Bulletin, 112(1), 155.

Funder, D. C., \& Ozer, D. J. (2019). Evaluating Effect Size in Psychological Research: Sense and Nonsense. Advances in Methods and Practices in Psychological Science, 2(2), 156168. https://doi.org/10.1177/2515245919847202

Gignac, G. E., \& Szodorai, E. T. (2016a). Effect size guidelines for individual differences researchers. Personality and Individual Differences, 102(November 2016), 74-78. https://doi.org/10.1016/j.paid.2016.06.069

Gignac, G. E., \& Szodorai, E. T. (2016b). Effect size guidelines for individual differences researchers. Personality and Individual Differences, 102, 74-78. https://doi.org/10.1016/j.paid.2016.06.069

Hofman, J. M., Goldstein, D. G., \& Hullman, J. (2020). How visualizing inferential uncertainty can mislead readers about treatment effects in scientific results. 12.

Reynolds, J. P., Pilling, M., \& Marteau, T. M. (2018). Communicating quantitative evidence of policy effectiveness and support for the policy: Three experimental studies. Social Science and Medicine, 218(October), 1-12. https://doi.org/10.1016/j.socscimed.2018.09.037

Reynolds, J. P., Stautz, K., Pilling, M., van der Linden, S., \& Marteau, T. M. (2020). Communicating the effectiveness and ineffectiveness of government policies and their impact on public support: A systematic review with meta-analysis. Royal Society Open Science, 7(1). https://doi.org/10.1098/rsos.190522

Wilkinson, L., \& American Psychological Association Task Force on Statistical Inference, T. (1999). Statistical methods on psychology journals: Guidelines and explanations. American Psychologist, 54, 594-604. 
